\title{
UK Renal Registry 11th Annual Report (December 2008): Appendix B Definitions and analysis criteria
}

\section{B:1 Definition of the take-on (incident) population}

The take-on population is defined as all patients over 18 who started RRT at UK Registry centres and did not have a recovery code within 90 days.

The treatment timeline is used to define take-on patients as follows:

If a patient has timeline entries from more than one centre then these are all combined and sorted by date. Then, the first treatment entry gives the first date of when they were receiving RRT. This is defined as a 'start date'. However, in the following situations there is evidence that the patient was already receiving RRT before this 'start date' and these people are not classed as take-on patients:

- Patients with an initial entry on the timeline of transferred in (codes 39 to 72 )

- those with an initial entry of transferred out (code 38)

- those with an initial treatment of lost to follow up (code 95)

- those who had graft acute rejection (code 31) and did not have a transplant on the same day

- those with an initial entry of transfer to adult nephrology (code 37)

- those with an initial entry of graft functioning (code 72)

- those with an initial entry of nephrectomy transplant (code 76)

Where none of these applies, the entry is defined as a take-on (as long as there is no recovery code within 90 days).

If there is a recovery code after 90 days then the program looks at the modality codes after this date to see if the patient restarted RRT. If they did then this is classed as another take-on (however long the gap between the recovery code and the next treatment entry).

For example, a patient may start RRT in 2005, recover and then restart RRT in 2005. Providing that they do not have a recovery code within 90 days on either occasion, such patients will be counted twice.

Note: patients restarting dialysis after a failed transplant were not counted as take-ons.

\section{B:2 Definition of the prevalent population for each year}

The prevalent population for a year is defined as all RRT patients over 18 , being treated at centres which were UK Registry centres for that year, who were alive on 31 December. It includes both incident patients for that year and patients who have been on treatment for longer. Note that any patients over 18 who are still being treated at paediatric centres are excluded.

Patients were only included under their primary treatment centre.

Patients who had transferred out, recovered function, stopped treatment without recovery of function or been lost to follow up before the end of the quarter were excluded.

\section{Further exclusions when analysing quarterly}

biochemistry or BP data

For these analyses, further restrictions were made to the prevalent cohort for each quarter:

Patients who had 'transferred in' to the centre in that particular quarter were excluded.
(C) $2009 \mathrm{~S}$. Karger AG, Basel

$1160-2110 / 09 / 1115-0293 \$ 26.00 / 0$

Accessible online at: www.karger.com/nec
UK Renal Registry, Southmead Hospital, Southmead Road, Bristol BS10 5NB, UK

Email: renalreg@renalreg.com 
Patients who had changed treatment modality in that particular quarter were excluded.

Patients who had been on RRT for less than 90 days were excluded.

Note: the length of time on RRT is calculated from the most recent take on date. So if a patient starts, then recovers, and then starts again this second start date is used. Also, for patients who are not defined as take on patients because their start date is unknown (for example, if their first timeline entry is a transfer in code) it is assumed that they have been on RRT for longer than 90 days and are included for every quarter.

\section{B:3 Statistical definitions}

\section{Death rate calculation}

A death rate per 100 patient years is calculated by counting the number of deaths and dividing by the person years exposed. This includes all patients, including those who died within the first 3 months of therapy. The person years at risk are calculated by adding up, for each patient, the number of days at risk (until they died or transferred out) and dividing by 365 .

\section{Odds ratio}

This is the odds of an event in one group divided by the odds in a reference group. For example, if the event is death (within a certain time) and you are comparing phosphate groups then for phosphate group 1.8 to $2.1 \mathrm{mmol} / \mathrm{L}$ the odds of the event are:

(probability of dying for someone with a phosphate of $1.8-2.1 \mathrm{mmol} / \mathrm{L}$ )

(probability of surviving for someone with a phosphate of $1.8-2.1 \mathrm{mmol} / \mathrm{L}$ )

The odds ratio is then:

(odds of dying if phosphate $1.8-2.1 \mathrm{mmol} / \mathrm{L}$ ) (odds of dying for reference group)

Note that when the event being analysed is death, often the odds ratio would not be used but a 'survival analysis' used instead. This takes into account the time when the event occurs and also allows for censoring (for example if people are lost to follow up). Such an analysis gives hazard ratios (see below) rather than odds ratios.

\section{Hazard function}

The hazard function is the probability of dying in a short time interval, conditional on survival up to that point.
Hazard ratio

For the same example as above, the hazard ratio is the:

(probability of dying in the next interval for a phosphate of $1.8-2.1 \mathrm{mmol} / \mathrm{L}$ )

(probability of dying in the next interval for a phosphate in the reference range)

Relative hazard

Following the notation of Collett, D. (2003): Modelling survival data in medical research, Chapman \& Hall, p. 57:

$$
h_{i}(t)=\exp \left(\beta x_{i}\right) \times h_{0}(t)
$$

The relative hazard is the $\exp \left(\beta x_{i}\right)$ component in the general proportional hazards model with age, the variable of interest and it's square as covariates. The plots were done for $\exp \left(\beta x_{i}\right)$ for different values of the variable of interest only, in other words, age was taken as a constant value of zero.

\section{B:4 General and modality definitions}

Definitions of analysis quarters

\begin{tabular}{ll}
\hline Quarter & Dates \\
\hline 1 & 1 January-31 March \\
2 & 1 April-30 June \\
3 & 1 July-30 September \\
4 & 1 October-31 December \\
\hline
\end{tabular}

The quarterly biochemistry data were extracted from renal centre systems as the last data item stored for that quarter. If the patient treatment modality was haemodialysis, the software will try to select a predialysis value.

\section{Home haemodialysis}

Home haemodialysis patients cease to be classed as such if they need longer than two weeks of hospital dialysis when not an in-patient.

\section{Satellite dialysis unit}

A renal satellite unit is defined as a haemodialysis facility that is linked to a main renal centre, is not autonomous for medical decisions and provides chronic outpatient maintenance haemodialysis but with no acute or in-patient nephrology beds on site. 
Start of established renal failure

Established renal failure (also known as end stage renal failure or end stage renal disease) was defined as the date of the first dialysis (or of pre-emptive transplant).

If a patient started as 'acute' renal failure and did not recover, the date of start of renal replacement should have been backdated to the start of acute dialysis. Many nephrologists do not do this and where this period of acute dialysis has been recorded in local systems, the UKRR will use this data to backdate the start of RRT.

If a patient was started on dialysis and dialysis was temporarily stopped for less than 90 days for any reason (including access failure and awaiting the formation of further access) except the recovery of renal function, the date of start of renal replacement therapy (RRT) in UKRR analyses remained the date of first dialysis.

\section{Change of modality from $P D$ to $H D$}

Sites are requested to $\log$ in their timeline changes from PD to HD if the modality switch is for longer than 30 days.

\section{B:5 Comorbidity definitions}

\section{Angina}

History of chest pain on exercise with or without ECG changes, ETT, radionucleotide imaging or angiography.

\section{Previous MI within last 3 months}

Detection of rise and/or fall of a biomarker (CK, CKMB or Troponin) with at least one value above the 99th percentile together with evidence of myocardial ischaemia with at least one of either:

a. ischaemic symptoms,

b. ECG changes indicative of new ischaemia (new ST$\mathrm{T}$ changes or new left bundle branch block),

c. development of pathological Q waves,

d. imaging evidence of new loss of viable myocardium or new regional wall motion abnormality.

This definition is from the European Society of Cardiology and American College of Cardiology.

Previous $M I>3$ months ago

Any previous MI at least 3 months prior to start of renal replacement therapy.

Statistical methodology and definitions

\section{Previous $C A B G$ or coronary angioplasty}

Previous episode of heart failure

Whether or not due to fluid overload.

\section{Cerebrovascular disease}

Any history of strokes (whatever cause) and including transient ischaemic attacks caused by carotid disease.

\section{Diabetes (not causing ESRF)}

This includes diet controlled diabetics.

\section{Chronic obstructive pulmonary disease}

Chronic obstructive pulmonary disease (COPD) is characterised by airflow obstruction. The airflow obstruction is usually progressive, not fully reversible and does not change markedly over several months.

- Airflow obstruction is defined as a reduced FEV1 (forced expiratory volume in 1 second) and a reduced FEV1/FVC ratio (where FVC is forced vital capacity), such that FEV1 is less than $80 \%$ predicted and FEV1/FVC is less than 0.7.

- The airflow obstruction is due to a combination of airway and parenchymal damage.

- The damage is the result of chronic inflammation that differs from that seen in asthma and which is usually the result of tobacco smoke.

There is no single diagnostic test for COPD. Making a diagnosis relies on clinical judgement based on a combination of history, (exertional breathlessness, chronic cough, regular sputum production, frequent winter 'bronchitis', wheeze) physical examination and confirmation of the presence of airflow obstruction using spirometry, (source: British Thoracic Society guidelines).

\section{Liver Disease}

Persistent enzyme evidence of hepatic dysfunction or biospy evidence or HbeAg or hepatitis C antigen (polymerase chain reaction) positive serology.

\section{Malignancy}

Defined as any history of malignancy (even if curative) e.g. removal of melanoma, excludes basal cell carcinoma.

\section{Claudication}

Current claudication based on a history, with or without Doppler or angiographic evidence. 
Ischaemic/neuropathic ulcers

Current presence of these ulcers.

Angioplasty, stenting, vascular graft (all non coronary) This category now includes vascular grafts (e.g. aortic bifurcation graft) and renal artery stents.
Amputation for peripheral vascular disease

\section{Smoking}

Current smoker or history of smoking within the last year. 